\title{
Characterization and expression analysis of the cytochrome bd oxidase operon from Desulfovibrio gigas
}

Servicos $9=$ Print $x$-mail Add to Marked List Save to EndNote Web

Servicos 9 क

Author(s): Machado P, Felix R, Rodrigues R, Oliveira S, Rodrigues-Pousada C

Source: CURRENT MICROBIOLOGY Volume: 52 Issue: 4 Pages: 274-281 Published: APR 2006

Times Cited: 3 References: 45 ExGitation Map beta

Abstract: Although classified as anaerobic, Desulfovibrio gigas contains a functional canonical membrane respiratory chain, including a cytochrome bd quinol oxidase as its terminal element. In the present study, we report the identification of the operon cydAB encoding the two subunits of cytochrome bd from this bacterium. Two hypothetical promoter regions and sequences resembling transcriptional regulators-binding sites have been identified. Amino acid sequence analysis revealed a high similarity to cytochrome bd from other organisms, presenting the conserved residues typical from these proteins. Reverse transcription polymerase chain reaction (RT-PCR) and Northern blot analysis confirmed the operon transcription. Gene expression was assessed by real-time RT-PCR in cells grown in different media and under exposure to oxygen and nitric oxide. mRNA levels were slightly enhanced in the presence of $150 \mathrm{mu} \mathrm{M} \mathrm{NO}$. However, in the presence of $10 \mathrm{mu} \mathrm{M} \mathrm{NO}$, a decrease was observed of the steady-state population of cydAB mRNA. No considerable effect was observed in the presence of fumarate/sulfate medium, $60 \mathrm{mu} \mathrm{M} \mathrm{O}-2$ or $10 \mathrm{mu} \mathrm{M}$ NO.

Document Type: Article 